\title{
Absence of magnetic evidence for superconductivity in hydrides under high pressure
}

\author{
J. E. Hirsch ${ }^{a}$ and F. Marsiglio ${ }^{b}$ \\ ${ }^{a}$ Department of Physics, University of California, San Diego, La Jolla, CA 92093-0319 \\ ${ }^{b}$ Department of Physics, University of Alberta, Edmonton, Alberta, Canada T6G 2E1
}

\begin{abstract}
It is generally believed that magnetization measurements on sulfur hydride under high pressure performed in 2015 [1] provided "final convincing evidence of superconductivity" [2] in that material, in agreement with theoretical predictions $[3,4]$. Supported by this precedent, drops in resistance that were later observed in several other hydrides under high pressure [2, 5] have been generally accepted as evidence of superconductivity without corroborating evidence from magnetic measurements. In this paper we challenge the original interpretation that the magnetic measurements on sulfur hydride performed in 2015 were evidence of superconductivity. We point out that a large paramagnetic contribution to the magnetic susceptibility was detected below $T_{c}$ and argue that its temperature dependence rules out the possibility that it would be a background signal; instead the temperature dependence indicates that the paramagnetic behavior originated in the sample. We discuss possible explanations for this remarkable behavior and conclude that standard superconductors would not show such behavior. We also survey all the other published data from magnetic measurements on this class of materials and conclude that they do not provide strong evidence for superconductivity. Consequently, we call into question the generally accepted view that conventional superconductivity in hydrogen-rich materials at high temperature and pressure is a reality, and discuss the implications if it is not.
\end{abstract}

PACS numbers:

\section{INTRODUCTION}

High-temperature conventional superconductivity has reportedly been found in several hydrogen-rich materials under high pressure in recent years [2, 5]. This behavior is predicted by calculations based on the conventional theory of superconductivity [2, 5-7]. The first material where this phenomenon was reportedly observed was sulfur hydride under pressures above 100GPa [1], reaching a critical temperature of $203 \mathrm{~K}$ for pressure $155 \mathrm{GPa}$. In this paper we analyze the magnetic evidence on which that claim was based. We argue that it indicates that the material is either a non-standard superconductor [8-10] or more likely is not a superconductor.

More generally, in recent work we [8, 9] and others [11] have called into question the interpretation that resistance drops measured in several high pressure hydrides are evidence of superconductivity. In a carbonaceous sulfur hydride claimed to become superconducting at room temperature [12], the resistance versus temperature data show extremely sharp drops, uncharacteristic of hightemperature superconductivity [8, 11]. Even more unusual is the fact that under application of a magnetic field the drops in resistance remain equally sharp. That is not the behaviour expected from standard superconductors, whether conventional or unconventional [13]. We furthermore pointed out that similar unphysical behaviour is observed in several other hydrides claimed to become superconducting under high pressure. We proposed a new category of 'nonstandard superconductors' to describe superconducting materials with such unusual behavior, and raised the possibility that the behavior observed is not due to superconductivity [9].

In this paper we focus on magnetic evidence of super- conductivity in hydrides under high pressure. We start by analyzing the claim that the magnetization data for sulfur hydride reported in 2015 [1] demonstrated that sulfur hydride under high pressure is a high-temperature superconductor.

\section{MAGNETIZATION OF SULFUR HYDRIDE}

Figure 1 shows the magnetization versus magnetic field data reported in Ref. [1] (see also [14]) from which it is inferred that the material is a superconductor below $203 \mathrm{~K}$. It is argued in Refs. [1, 14] that the graphs show the diamagnetic response of the superconductor superposed to a paramagnetic signal originating in the diamond anvil cell (DAC) where the sample resides.

However, from the data shown in Fig. 1 we can extract the paramagnetic contribution to the magnetization. We plot the corresponding magnetic susceptibility $\chi=M / H$, with $\mathrm{M}$ the magnetization and $\mathrm{H}$ the applied field) versus temperature in Fig. 2.

Fig. 2 shows that the paramagnetic contribution goes to zero, or very close to zero, at or around the inferred superconducting critical temperature of the sample, $203 \mathrm{~K}$. The three data points shown in the figure at temperatures above $200 \mathrm{~K}$ were obtained from Fig. $4 \mathrm{~d}$ of Ref. [1]. If the paramagnetic contribution resulted from a magnetic background signal in the DAC, we would expect it to follow a Curie-Weiss or Curie law. In Fig. 2 we have fitted such laws to the measured values of the paramagnetic susceptibility at $50 \mathrm{~K}$ and $200 \mathrm{~K}$. It can be seen that neither of them fits the observed behavior.

Let us consider for comparison similar data obtained for the superconductor $\mathrm{GdBa}_{2} \mathrm{Cu}_{3} \mathrm{O}_{7}$ in Ref. [16], shown in Fig. 3. This paper was cited in Ref. [1] as giving anal- 


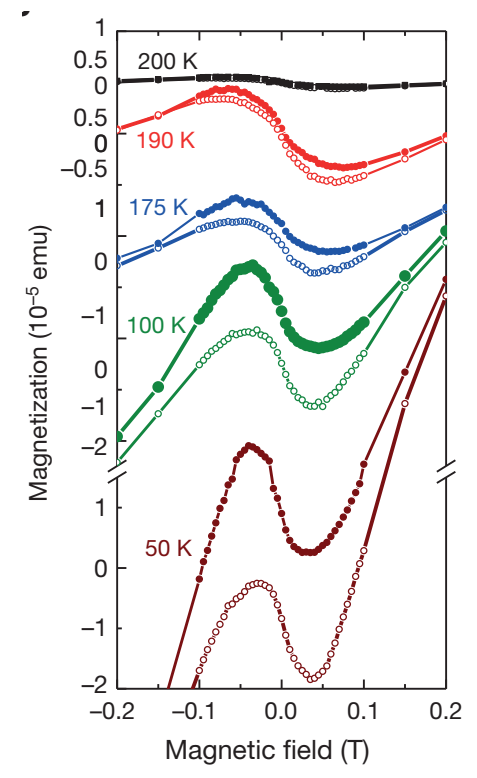

FIG. 1: Magnetization measurements for sulfur hydride at a pressure of $155 \mathrm{GPa}$ from Fig. 4c of ref. [1]. The open (closed) circles correspond to magnetic field increasing (decreasing).

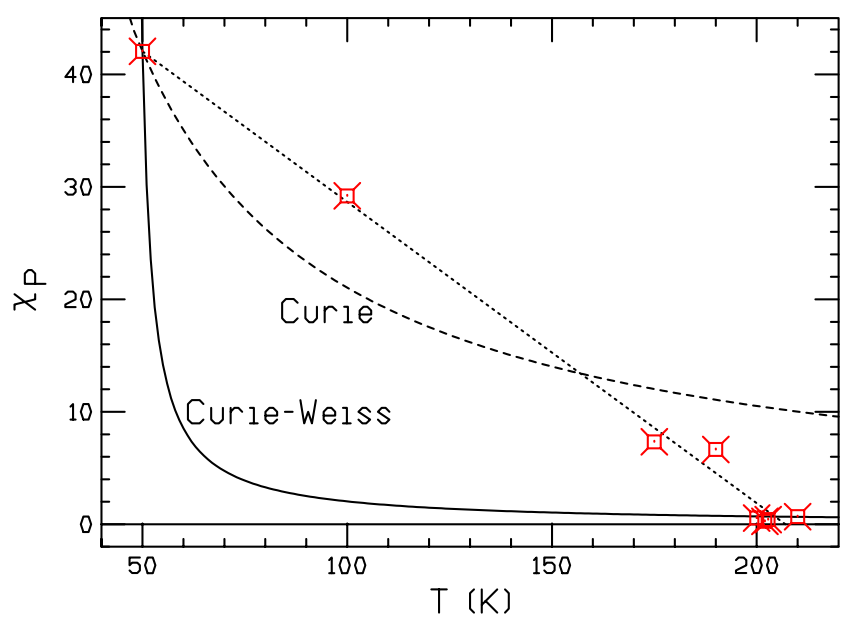

FIG. 2: Paramagnetic contribution to the susceptibility $\chi_{P}$ inferred from Fig. 1, in units $10^{-5} \mathrm{emu} / \mathrm{T}$. The experimental data are the points, the dotted line is a guide to the eye.

ogous behavior to the one shown in Fig. 1 due to the presence of the magnetic $G d$ atoms.

Indeed, we see a similarity in the magnetization data of Fig. 3 and Fig. 1: a diamagnetic signal for small fields is superposed with a paramagnetic signal that persists to much larger fields. Following the same procedure as in obtaining Fig. 2 from Fig. 1, we show in Fig. 4 the paramagnetic contribution to the susceptibility inferred from the data in Fig. 3.

It can be seen that a simple Curie law fits the paramagnetic susceptibility data inferred from Fig. 3 quite well, in contrast to the situation in Fig. 2. Therefore, it is completely plausible that the diamagnetic part of

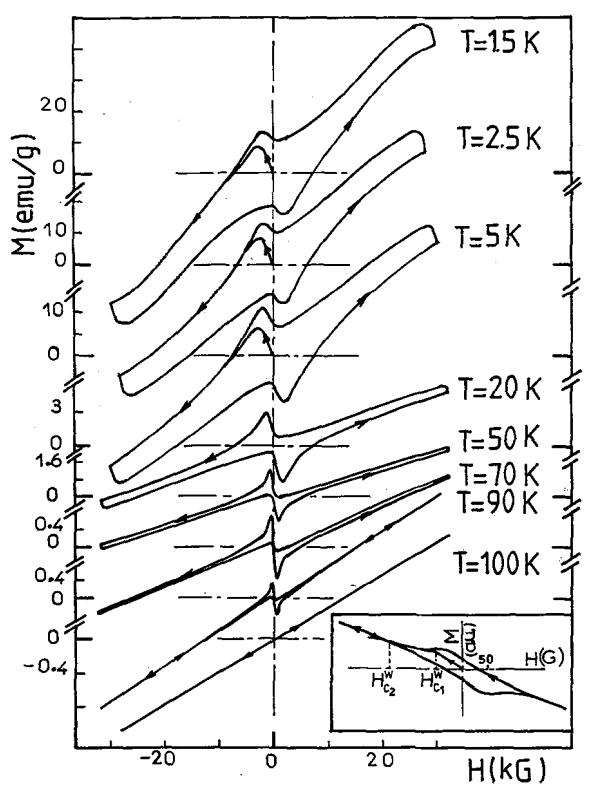

FIG. 3: Magnetization measurements for $\mathrm{GdBa}_{2} \mathrm{Cu}_{3} \mathrm{O}_{7}$ from Fig. 1 of Ref. [16].

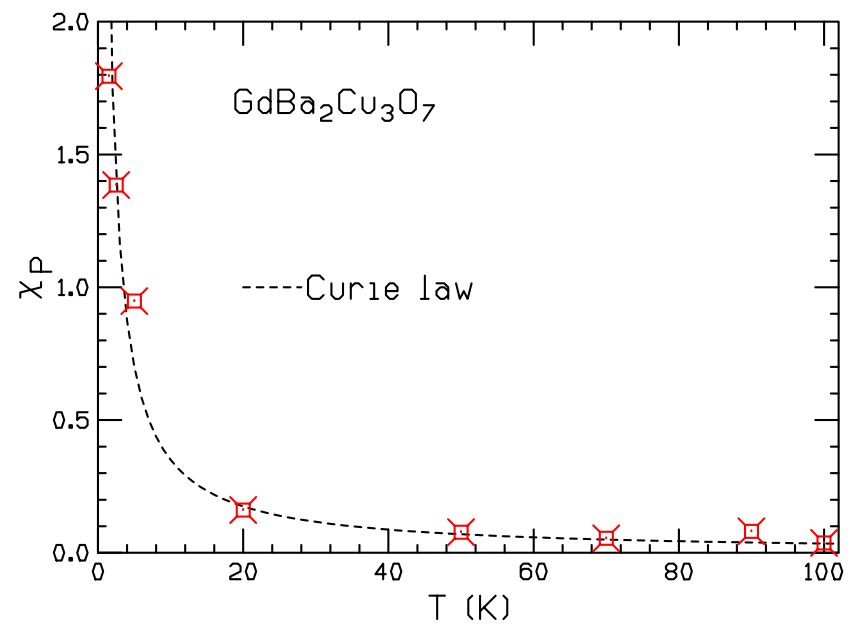

FIG. 4: Paramagnetic contribution to the susceptibility $\chi_{P}$ inferred from Fig. 3, in units emu/g/kG. The experimental data are the points.

the response in Fig. 3 originates in superconductivity while the paramagnetic part originates in the large magnetic moment of $G d$ atoms that behave essentially as isolated magnetic impurities. A more detailed analysis performed in Ref. [16] yields a Curie-Weiss temperature $\theta \sim-2 \pm 1 \mathrm{~K}$.

We argue that the explanation proposed by the authors of [1] for the behavior of the magnetization displayed in Fig. 1 is completely implausible. The key point is that there would be no reason for the paramagnetic contribution to the susceptibility originating in a background signal to go to zero at essentially the same temperature at which the diamagnetic contribution from the sample goes 
to zero, as Fig. 2 indicates. Instead, the fact that it does, indicates that the paramagnetic contribution and the diamagnetic contribution originate from the same physical object, presumably the sample.

We then have to ask: is there any other standard superconductor where such a behavior of the magnetic susceptibility below $T_{c}$ is observed? We define "standard superconductor' theoretically by superconductors described in Tinkham's book [13], and experimentally by superconductors belonging to any of the 32 families of conventional, unconventional and possibly unconventional superconductors surveyed in Ref. [15], that cover all superconducting materials known in 2015. In Ref. [15], materials were classified as 'conventional' (classes C1 to C12), 'unconventional' (classes U1 to U11) and 'possibly unconventional' (classes P1 to P9).

Among these superconducting material classes there are the conventional magnetic superconductors (C10) and the ferromagnetic superconductors (U7), that one could consider as prime candidates for exhibiting large paramagnetic susceptibility below $T_{c}$ superposed to a diamagnetic response. One may also consider numerous other classes that exhibit magnetic phases nearby in the phase boundary such as Chevel phases (C9), borocarbides (P3), plutonium compounds (P4), heavy-fermion superconductors (U1), organic compounds (U2), cuprate superconductors (U3, U4), strontium ruthenate (U5), cobalt oxyde hydrate (U8), iron-based superconductors (U10, U11). However, none of these materials exhibit the anomalous behavior of magnetic susceptibility below $T_{c}$ exhibited by sulfur hydride shown in Fig. 1.

Regarding theory, Tinkham's book describes the phenomenology of the magnetic behavior of standard type I and type II superconductors expected from London theory and Ginzburg-Landau theory, irrespective of the particular microscopic mechanism giving rise to superconductivity [13]. That physics does not give rise to the behavior of magnetization versus field shown in Fig. 1.

\section{NEW PHYSICS OR EXPERIMENTAL ARTIFACT?}

Forgetting for the moment about superconductivity, we know of no magnetic material that would show a behavior of its magnetic susceptibility like the one shown in Fig. 2.

The magnetic response of materials originates in orbital magnetism or spin magnetism or a combination of both. Let us recall the known behaviours:

1) Temperature independent paramagnetic susceptibility is seen in metals due to the electron magnetic moment and Fermi statistics.

2) Small temperature independent diamagnetic susceptibility is seen in insulators with atoms with closed shells.

3) Temperature dependent paramagnetic susceptibility originates in localized magnetic moments or in the magnetic moment of itinerant electrons in magnetic met- als, and results in Curie or Curie-Weiss behavior with a Curie-Weiss temperature $\theta$. If $\theta$ is positive the material undergoes a transition to a ferromagnetic state at $T=\theta$. If $\theta$ is negative the material may undergo a transition to an antiferromagnetic state at a finite temperature.

The magnetic susceptibility shown in Fig. 2 increases by a factor of over 60 from $200 \mathrm{~K}$ to $50 \mathrm{~K}$, i.e. in a temperature range where the absolute temperature decreases by a factor of 4 . We know of no other way that this could happen other than the system undergoing a transition to a ferromagnetic state at temperature slightly below $50 \mathrm{~K}$ (the fit shown in Fig. 2 assumed a Curie-Weiss temperature $\theta=47.5 \mathrm{~K})$. However it is impossible to fit the susceptibility data at temperatures in-between $50 \mathrm{~K}$ and $200 \mathrm{~K}$ with such behavior, as seen in Fig. 2.

Within mean field theory, the critical temperature for onset of ferromagnetic order in a system of magnetic moments interacting through a ferromagnetic coupling $J$ can be written in the form

$$
T_{c}=\frac{z J}{k_{B}}
$$

where $\mathrm{z}$ is the number of nearest neighbors to a moment and $k_{B}$ is Boltzmann constant. The magnetic susceptibility is given by (Curie-Weiss law)

$$
\chi_{P}(T)=\frac{\chi_{0}}{T / T_{c}(T)-1}
$$

with $\chi_{0}$ a constant. To describe the behavior shown in Fig. 2 we would have to assume that $T_{c}$ in Eq. (2) is itself a function of temperature, as indicated in Eq. (2), smaller than $T$ and decreasing as $T$ decreases with increasingly smaller $\left(T-T_{c}(T)\right)$, so as to give the approximately linear behavior shown in Fig. 2. This could result from a temperature dependent $z(T)$ or $J(T)$ in Eq. (1) decreasing as $T$ decreases. However we know of no physical mechanism that would give rise to such behavior.

There have been suggestions in the literature that hydrogen or hydrogen-rich compounds under high pressure would undergo a transition to a magnetic phase at low temperatures rather than a superconducting phase $[17,18]$. Such models would possibly explain the large paramagnetic response seen at low temperatures, but are unlikely to yield the peculiar linear temperature dependence of the magnetic susceptibility shown in Fig. 2.

Considering now the superconductivity, note that the magnetic field range in Fig. 1 is a very small fraction of the assumed upper critical field in this material, approximately $70 \mathrm{~T}$ [1]. Nonetheless, since the field values are likely higher than $H_{c 1}$, we assume the system is in the mixed state, with normal vortex cores of radius $\xi$, the coherence length. and the magnetic field screened over a radius $\lambda_{L}$, the London penetration depth, around each vortex. The total magnetic field is given by

$$
B=H+4 \pi M
$$

where $H$ is the applied field and $M$ the magnetization. Since each vortex can hold exactly one quantum of flux 
$\phi_{0}$, as the temperature is lowered the number of vortices must increase to accommodate the extra magnetic flux resulting from the induced magnetization (in the range where the sample is in the mixed state). This should cause the diamagnetic response to decrease as the temperature is lowered rather than increase rapidly as indicated by the data in Fig. 1.

In summary, we cannot imagine a plausible explanation for the reported linear increase in paramagnetic response as the temperature is lowered within the conventional understanding of magnetism in solids, and the joint increase in paramagnetic and diamagnetic response as the temperature is lowered shown in Fig. 1 appears to contradict everything we know about superconductivity in standard superconductors. Furthermore we have argued that it is not plausible that the paramagnetic response originates in a different physical region of the apparatus used in the experiment, given the fact that it goes essentially to zero at the same temperature where the diamagnetism disappears.

We conclude that it is highly likely that the experimental data shown in Fig. 1 that were reported in Ref. [1] are an experimental artifact and do not reflect real physics.

\section{OTHER MAGNETIC EVIDENCE FOR SUPERCONDUCTIVITY IN HYDRIDES UNDER HIGH PRESSURE}

Since 2015, superconductivity in high pressure hydrides evidenced by resistance measurements in diamond anvil cells has been reported in the following compounds: $S H_{x}$ up to $203 K[1,19], P H_{x}$ above $100 \mathrm{~K}$ [20], $\mathrm{LaH}_{x}$ at $250 \mathrm{~K}$ [21, 22], above $260 \mathrm{~K}$ [23] and above 550K [24], $Y H_{x}$ at $243 \mathrm{~K}$ [25-27], $T h H_{x}$ at $161 \mathrm{~K}$ [28], $\mathrm{Pr} H_{x}$ at $9 \mathrm{~K}$ [29], $\mathrm{LaYH}_{x}$ at 253K [30], CSH at room temperature [12], $\mathrm{CeH}_{x}$ above $120 \mathrm{~K}$ [31], $\mathrm{SnH}_{x}$ at $70 \mathrm{~K}$ [32], $\mathrm{BaH}_{x}$ around 20K [33], and $\mathrm{CaH}_{x}$ around $215 \mathrm{~K}$ [34, 35]. That is 19 different reports on 12 different materials. These papers have been cited 2,450 times according to Google Scholar $(2 / 24 / 21)$, with essentially all citing papers assuming that superconductivity in these materials is an established fact. Numerous news media have also informed the general public that superconductivity in these materials is an established fact.

However, to establish that a material is a superconductor it is essential to show that it exhibits a response to magnetic fields expected for superconductors. Many of the papers cited above limit themselves to consider the effect of magnetic field on resistance. We will discuss that effect at the end of this section. However, concerning the magnetic response to magnetic fields, we have reviewed the existing literature on high pressure superconductivity in hydrides looking for such information and have found only 5 papers, on three different materials, namely:

- The magnetization measurements on sulfur hydride [1] discussed in the previous sections.
- Ac magnetic susceptibility measurements for $C S H$ [12].

- Ac magnetic susceptibility measurements for sulfur hydride [36].

- Ac magnetic susceptibility measurements for $L a H_{x}$ [37].

- Nuclear resonant scattering (NRS) measurements for sulfur hydride [38].

Let us consider each of these.

\section{A. Magnetization of sulfur hydride}

As discussed in the previous sections, the magnetization versus magnetic field data given in [1] shown in Fig. 1 of this paper are not consistent with the behavior of standard superconductors and magnetic materials. The paper [1] also plots magnetization versus temperature in its Fig. 4a, which we reproduce in Fig. 5, showing that it increases sharply from approximately $-4 \times 10^{-7} \mathrm{emu}$ to $+7 \times 10^{-7}$ emu as the temperature is raised across $200 \mathrm{~K}$.

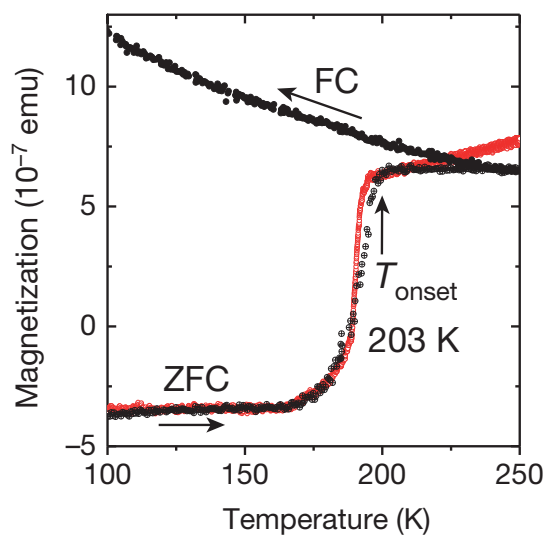

FIG. 5: Magnetization versus temperature reported in Ref. [1] (Fig. 4(a)), for field cooling (FC) and zero field cooling (ZFC), or more accurately, field heating. The red dots are resistance data "scaled and moved vertically to compare with the magnetization data" [1].

However, in their extended data Figure 6 the authors show that there is a 'background' magnetization signal that is two orders of magnitude larger than the signal shown in Fig. 5. As explained in the figure caption of extended data Figure 6, a background signal was subtracted in order to plot the data in Fig. 5. However because there is no precise way to tell what part of the raw signal is 'background', it means there is no way to know whether the sample signal actually does change sign from negative to positive as the temperature is raised, as Fig. 5 purports to show. In fact, the measured data did not provide information that the signal below $200 \mathrm{~K}$ is diamagnetic and above $200 \mathrm{~K}$ paramagnetic, and therefore 

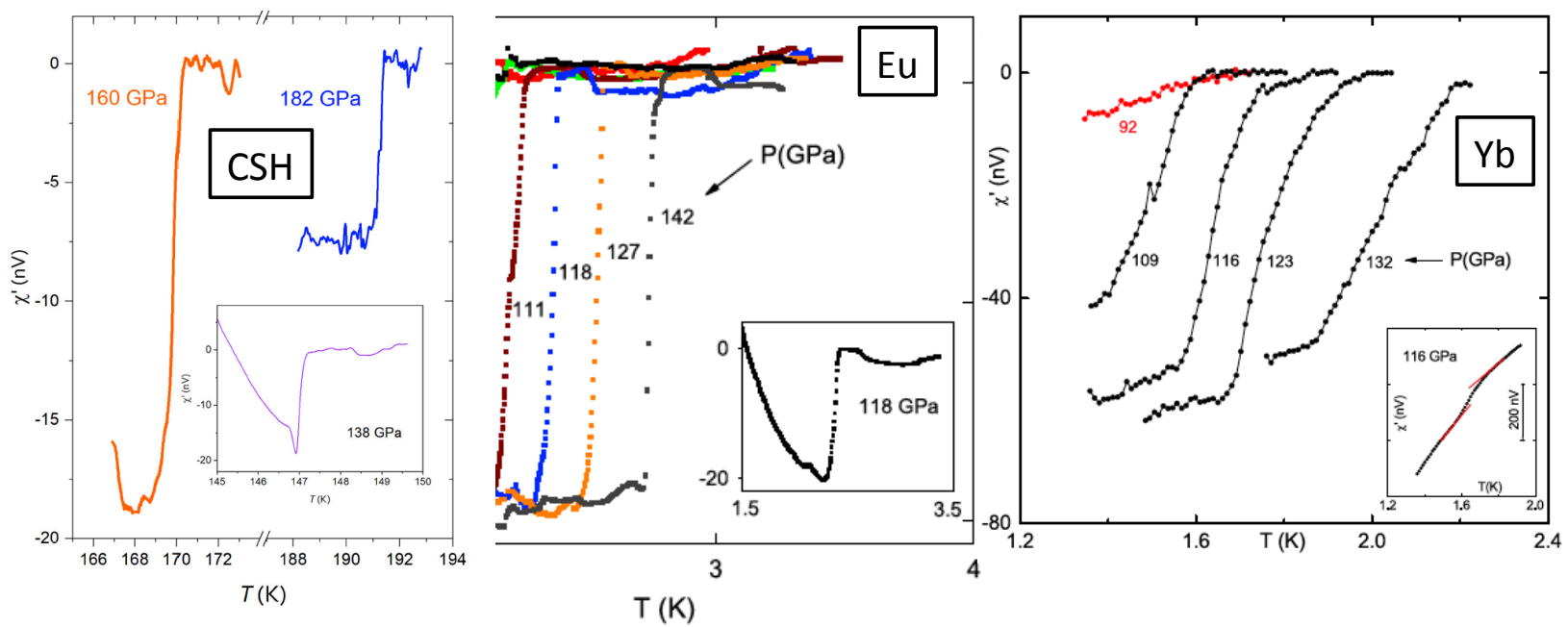

FIG. 6: Ac magnetic susceptibility for three materials under pressure in DAC's: CSH ([12]), and the elements Eu ([40]) and $\mathrm{Yb}([41])$. The insets show the raw data, from which the signal shown is extracted by substraction of a background signal.

the authors' statement in reference to their fig. 4(a) "a sharp transition from the diamagnetic to the paramagnetic state (Fig. 4a) was observed for zero-field-cooled (ZFC) material" is profoundly misleading. The reality is that there was no evidence from the measured data that the low temperature state of the sample was diamagnetic.

The myth has persisted for the ensuing 5 years until the present. In a recent review paper [5] the authors (one of whom also coauthored [1]) state in reference to this figure "The ZFC curve demonstrates a rather sharp transition from the diamagnetic to the paramagnetic state, which classified as a superconducting one. The onset temperature is $T_{c}=203 \mathrm{~K} . "$

It should also be noted that the field cooled (FC) data shown in Fig. 5 show absolutely no signature of anything happening around $200 \mathrm{~K}$. This is also in contradiction with the behavior of standard superconductors, where typically a smaller but non-zero signal of the transition is seen also in FC measurements.

\section{B. Ac magnetic susceptibility for $\mathrm{CSH}$}

Reference [12] reports ac magnetic susceptibility measurements on a carbonaceous sulfur hydride (CSH) that show sharp drops at temperatures close to where the resistance versus temperature curves show drops. An example from Ref. [12] is shown on the left panel of Fig. 6 . We will compare this behavior with other such data for materials in diamond anvil cells under high pressure, namely the elements $\mathrm{Eu}$ [40] (middle panel) and $\mathrm{Yb}$ [41] (right panel).

As pointed out in Ref. [39] there is a problem with the data for both CSH and Eu. It is well known [42] that in these experiments there is a large background signal that needs to be substracted from the raw data to extract the susceptibility of the sample. In the right panel of Fig. 6, the inset shows raw data obtained for $\mathrm{Yb}$ under pressure by Song et al. [41], and the right panel shows the extracted signal. The raw data reflect a smooth linear background, and superposed to it a small drop that is plotted in the main right panel. These measurements together with the resistance measurements reported in [41] give clear and unambiguous evidence that $\mathrm{Yb}$ becomes superconducting in these experiments.

Instead, the raw data shown in the insets of the left and middle panels of Fig. 6, for both CSH and the element Eu show features that are inconsistent with superconductivity, namely: (i) the fact that the raw signal is flat for temperatures above the drop, and (ii) the fact that the raw signal rises steeply for temperatures below the drop. These data are qualitatively different from the featureless linear background seen in the right panel of Fig. 6 which is typical for these measurements. As discussed in Ref. [39], this indicates that both the data for CSH and $\mathrm{Eu}$ are either (a) an experimental artifact and do not reflect real physics, or (b) an indication that a magnetic transition instead of a superconducting transition is taking place, which could account for the steep rise in the raw susceptibility data at lower temperature. The initial drop could be attributed to the sample becoming more conducting as it develops magnetic order.

As noted in Ref. [39], the measurements of the left and middle panels of Fig. 6 were performed with the same equipment and by the same researcher, which supports the possibility that they could be due to an experimental artifact.

\section{Ac magnetic susceptibility for sulfur hydride}

Reference [36] (see also [43]) reports results of ac magnetic susceptibility measurements for sulfur hydride, that look qualitatively similar to the data for $\mathrm{CSH}$ reported in 


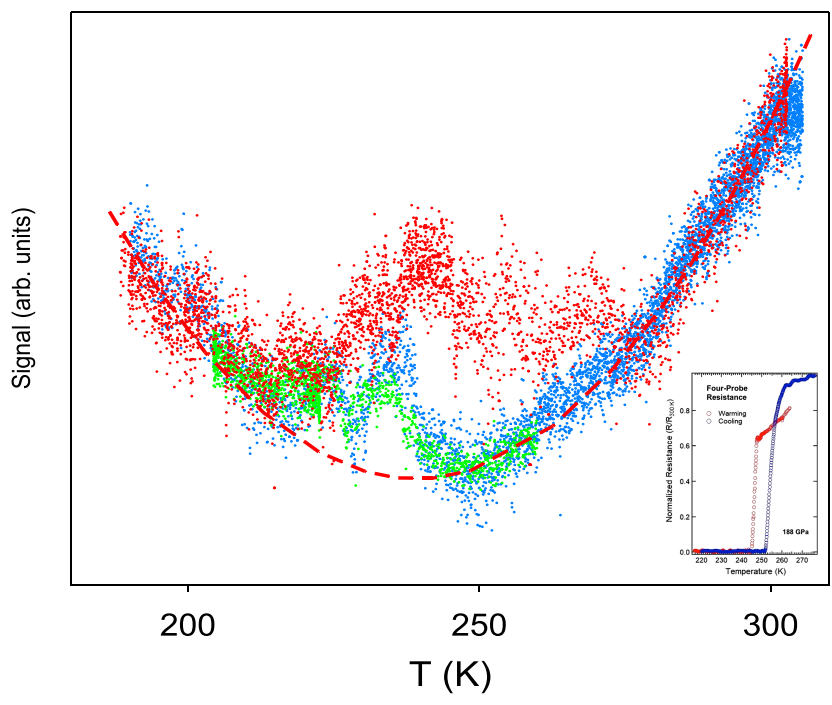

FIG. 7: Magnetic response signals for $L a H_{x}$ from Ref. [37]. The blue, green and red points correspond to pressures $164 \mathrm{GPa}, 169 \mathrm{GPa}$ and $180 \mathrm{GPa}$. The assumed background is shown by a red dashed line [37]. The inset shows resistance versus temperature results for this material, from Ref. [23].

[12], left panel of Fig. 6. However, Ref. [36] does not give essential information to allow the reader to evaluate the significance of the data presented. It states "The protocol of the magnetic-susceptibility technique for measuring the superconductivity is described in previous works" and cites three references, [44-46]. However, it does not tell the reader what frequency was used in the measurements, which is essential in order to understand the significance of the amplitude of the signal reported (the frequencies used in Refs. [44-46] are all different, as are the detailed procedures). Nor does Ref. [36] give any information on the background signal, nor does it inform the reader that a subtraction of background signal was performed, nor does it provide any raw data.

We have attempted to obtain additional information to evaluate the significance of these data by contacting the authors of Ref. [36] but did not receive any response. Given the limited information in the published paper, we argue that it cannot be taken as clear evidence for a superconducting transition in sulfur hydride.

\section{Ac magnetic susceptibility for lanthanum hydride}

Reference [37] reports ac magnetic susceptibility measurements on $\mathrm{LaH}_{x}$, using a low-frequency modulation technique discussed in Refs. [44, 45]. The data are shown in Fig. 7. This technique was not used in Ref. [36], despite the ambiguous statement in Ref. [36] quoted above that suggests otherwise. With this technique the evidence for superconductivity is detected as a peak in the signal rather than a drop. Fig. 7 shows very broad fea- tures, much broader than the width of the resistive transitions reported in [21-23] for this compound (see inset in Fig. 7). Note that their amplitude relative to the background cannot be ascertained since no vertical scale is given. Ref. [37] states "We were able to detect a measurable signal, however, the estimated sample size proved to be very small", and suggests the possible existence of multiple phases and pressure gradients to account for the broad signals. Note in contrast that in sulfur hydride the width of the transition was reported to be the same in magnetic and resistive measurements (Fig. 5, black points for ZFC and red points for resistance).

\section{E. Nuclear resonant scattering for sulfur hydride}

Reference [38] reports results of a nuclear resonance scattering (NRS) experiment as evidence that that sulfur hydride expels magnetic fields. In Ref. [10] we have carefully analyzed the data presented in Ref. [38]. Fig. 8 shows schematically the magnetic field configuration that Ref. [38] claims is detected in their NRS experiment, proving that the sample excludes magnetic fields. However, if this was true it would imply, given that the applied magnetic field was $0.68 T$, that the magnetic field at the edge of the sample was $2.5 \mathrm{~T}$ [10], hence that $H_{c 1}$, the lower critical field of this material, is larger than $2.5 \mathrm{~T}$ at temperatures below $50 \mathrm{~K}$, where it is claimed that the field is totally excluded [38]. This value of $H_{c 1}$ differs from the lower critical field for this material estimated in Ref. [1] from the magnetization data, $H_{c 1} \sim 0.003 T$, by three orders of magnitude. It is also more than two orders of magnitude larger than any lower critical field known for any other superconductor, and incompatible with other properties of the material as discussed in [10].

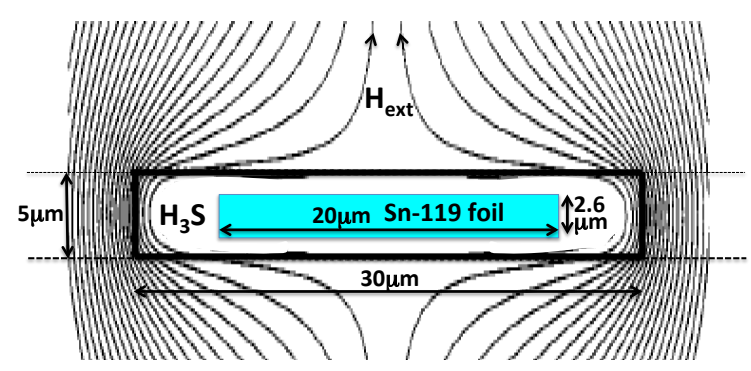

FIG. 8: Geometry of the NRS experiment with magnetic field perpendicular to the sensor film $[10,38]$. The applied magnetic field was $0.68 T$.

It should also be noted that such an experiment to detect the Meissner effect using nuclear resonance scattering has never been used, either before or after the experiment reported in [38], to detect the Meissner effect for this or any other superconducting material according to the published literature. 
The data surveyed above comprise the totality of measurements of the magnetic response of hydrides under high pressure to applied magnetic fields that exist in the scientific literature to date. We argue that they do not provide strong evidence for the existence of superconductivity in these materials, for the variety of reasons discussed above.

\section{F. Effect of magnetic field on resistance}

Most of the 19 papers cited above that report the finding of high temperature superconductivity in hydrides measured the effect of magnetic field on resistance, and found that a large magnetic field lowers the apparent transition temperature. From these measurements they extract values of the upper critical magnetic field $H_{c 2}$, in the range approximately between $50 T$ and $150 T$.

However, we have pointed out in Refs. [8, 9] that the resistance in the presence of magnetic field that was found often does not follow the behavior expected for normal superconductors, which is that the resistive transition broadens under application of a magnetic field. This led us to conclude [9] that either these materials are "nonstandard superconductors", with properties markedly different from those of standard conventional and unconventional superconductors, or more likely that they are not superconductors. If the latter, the reason that the apparent $T_{c}$ shifts downward when a magnetic field is applied would not be related to superconductivity.

To test this hypothesis, we propose that the effect of magnetic field on resistance as a function of the orientation of the magnetic field with respect to the sample should be explored. To our knowledge, all the experiments performed to date that measured resistance applied the magnetic field in the direction perpendicular to the plane of the sample; this plane is defined as perpendicular to the short dimension of the sample, which is parallel to the applied pressure. As shown in Fig. 8, the plane is horizontal. So in particular we suggest that the experiments should be repeated with the magnetic field applied in the plane of the sample.

We note that the lattice structures that have been hypothesized for these compounds are isotropic [2, 5, 37] and the pressure is assumed to be hydrostatic [21, 47], so that the upper critical field $H_{c 2}$ should be the same in directions perpendicular and parallel to the plane of the sample. Therefore, if the effect of the magnetic field on resistance is due to the onset of superconductivity, the temperature at which the resistance begins to drop should be independent of the orientation of applied magnetic field. The resistance below that temperature on the other hand may have some variation with orientation of magnetic field perpendicular and parallel to the plane as well as for the two orthogonal orientations possible within the plane.

Instead, if the drop of resistance is not due to superconductivity but for example to the action of the magnetic
Lorentz force on normal charge carriers giving rise to 'geometrical magnetoresistance' [48, 49] we would expect a much smaller effect of magnetic field parallel versus perpendicular to the plane. Or, if the drop in resistance is related to the system developing magnetic order [17, 18] in some particular direction, the effect of an applied magnetic field would be expected to depend on its orientation.

\section{DISCUSSION}

Electrical resistivity in materials that are not superconducting covers a range from about $10^{-8} \Omega m$ to $10^{20} \Omega m$, that is, 28 orders of magnitude. For that reason a drop of two or three orders of magnitude in the resistance of a material as a function of temperature or pressure, as typically reported for the hydrides, could be due to many reasons other than superconductivity. It should also be noted that papers in this field almost always show resistance rather than resistivity data due to the difficulty of obtaining reliable information on samples' dimensions. This precludes the possibility of evaluating the significance of the magnitude of the resistivity in these materials. It should also be recalled that throughout the history of superconductivity there have been many reports of 'USO's, "unidentified superconducting objects," based on observations of resistance drops, that were never confirmed [50]. Unlike what has been the tradition in the field of superconducting materials, for the particular class of hydrides under high pressure even drops of resistance versus temperature by less than an order of magnitude are interpreted as unmistakable signs of a superconducting transition [19, 22, 32].

To establish that a material is a superconductor it is essential to show that it exhibits the characteristic behavior in external magnetic fields that only superconducting materials do. As we have shown in this paper, such evidence for the hydrides is scant and ambiguous at best. Furthermore it is incomprehensible that such measurements once performed are not repeated in the same or in different labs with the same or higher accuracy to either confirm or rule out the initial conclusions. The 2015 measurements of magnetization in sulfur hydride discussed in this paper required the development of a new high pressure technique, a specially designed miniature nonmagnetic DAC cell made of $\mathrm{Cu}$-Ti alloy that could accomodate a SQUID magnetometer [2, 14]. Yet no results using this sophisticated apparatus other than the ones shown in Fig. 1, neither for sulfur hydride nor for any other hydride, have been reported in the ensuing 6 years to the present, during which the number of supposedly superconducting hydrides mushroomed from 1 to 11 . The nuclear resonant study that supposedly proved the Meissner effect for sulfur hydride in 2016 [38], discussed in Sect. IV and in Ref. [10], has never been repeated for any other superconductor, hydride or otherwise.

Based on the analysis of the magnetic evidence discussed in this paper, together with the anomalous re- 
sistance versus temperature behavior in several hydrides discussed in our recent work [8,9] and that of others [11], we conclude that the existence of high temperature superconductivity in hydrides under high pressure is not an established fact. This conclusion is in contradiction with the general consensus. So we may ask: why have most scientists, both those that work in the field and those that watch it from nearby, uncritically accepted the existence of high temperature superconductivity in pressurized hydrides as a fact, on the basis of flimsy experimental evidence?

Examination of the literature in this field suggests the reason for this anomalous state of affairs: the field is entirely driven by theory. Essentially every experimental paper reporting measurements interpreted as superconductivity in a hydride starts with a lengthy exposition of why the conventional BCS-Eliashberg-electron-phonon theory of superconductivity makes the existence of high temperature superconductivity in the material inevitable and unavoidable. Sometimes experimental evidence for superconductivity is only presented towards the end of the paper, almost as an afterthought [29]. In cases where the experiment doesn't find what the theory predicted, the experiment is not interpreted as casting doubt into the applicability of the theoretical framework used, instead a new twist is added to the theory to agree with the experimental observation, or it is concluded that 'the sample made a mistake' [51, 52]. Purely theoretical papers are written and titled in such a way that they appear to report the finding of a new superconducting material in real life when in actual fact it was only found in a computer [53-55].

If BCS-Eliashberg-electron-phonon theory is the correct theory to describe the superconductivity of a large set of materials in nature, the so-called conventional superconductors, such an approach may be justified. How- ever, it should be recalled that historically, conventional BCS-Eliashberg theory coupled with density-functional band structure calculations has not been an effective tool to predict and find new superconductors [57]. Are we to believe that among the 32 classes of superconducting materials surveyed in [15], the theory is singularly effective in predicting new superconducting materials $[2,5-7]$ in class $\mathrm{C} 7$, compressed hydrogen-rich materials [56], and not in the other 31 classes? Why would that be the case?

For the moment, unless or until the high temperature superconductivity of hydrides under pressure is convincingly established through experiments that reproducibly measure the magnetic response expected for superconductors, we suggest that an alternative possibility should be considered: that the high pressure hydrides may in fact not be high temperature superconductors; hence that BCS-Eliashberg theory may not be an effective tool to predict superconducting materials in any of the 32 classes of materials surveyed in [15]; hence that specifically the very definite prediction of the theory that high temperature superconductivity exists in pressurized hydrides may not be correct. If that is proven to be the case, if such a compelling prediction of the theory is proven wrong, the possibility that BCS-Eliashberg-electron-phonon theory may not correctly describe the physics of superconductivity of any superconducting material, as was proposed by one of us in Refs. [58, 59], should become compelling.

\section{Acknowledgments}

JEH is grateful to V. Struzhkin for a stimulating discussion. FM was supported in part by the Natural Sciences and Engineering Research Council of Canada (NSERC).
[1] A.P. Drozdov, M.I. Eremets, I. A.Troyan, V. Ksenofontov and S. I. Shylin, 'Conventional superconductivity at 203 kelvin at high pressures in the sulfur hydride system', Nature 525, 73-76 (2015).

[2] C. J. Pickard, I. Errea and M. I. Eremets, "Superconducting Hydrides Under Pressure", Ann. Rev. Cond. Matt. Phys. 11, pp 57-76 (2020) and references therein.

[3] Y. Li, J. Hao, Y. Li and Y. Ma, "The metallization and superconductivity of dense hydrogen sulfide", J. Chem. Phys. 140, 174712 (2014).

[4] D. Duan, Y. Liu, F. Tian, D. Li, X. Huang, Z. Zhao, H. Yu, B. Liu, W. Tian, and T. Cui, "Pressure-induced metallization of dense $\left(\mathrm{H}_{2} \mathrm{~S}\right)_{2} \mathrm{H}_{2}$ with high- $T_{c}$ superconductivity", Sci. Rep. 4, 6968 (2014).

[5] J. A. Flores-Livas et al., "A perspective on conventional high-temperature superconductors at high pressure: Methods and materials", Physics Reports 856, 1-78 (2020).

[6] L. Boeri and B. Bachelet, "Viewpoint: the road to roomtemperature conventional superconductivity", J. Phys.
Cond. Matt. 31, 234002 (2019).

[7] Y. Quan, S. S. Ghosh, and W. E. Pickett, "Compressed hydrides as metallic hydrogen superconductors", Phys. Rev. B 100, 184505 (2019).

[8] J. E. Hirsch and F. Marsiglio, "Absence of high temperature superconductivity in hydrides under pressure", arXiv:2010.10307 (2020).

[9] J. E. Hirsch and F. Marsiglio, "Nonstandard superconductivity or no superconductivity in hydrides under high pressure", arXiv:2012.12796 (2020)

[10] J. E. Hirsch and F. Marsiglio, "Meissner effect in nonstandard superconductors", arXiv:2101.01701 (2021).

[11] M. Dogan and M. L. Cohen, "Anomalous behavior in high-pressure carbonaceous sulfur hydride", arXiv:2012.10771v1 (2020).

[12] E. Snider et al., 'Room-temperature superconductivity in a carbonaceous sulfur hydride', Nature 586, 373 (2020).

[13] M. Tinkham, "Introduction to superconductivity", Second Edition, McGraw Hill, New York, 1996.

[14] A. Drozdov, "Superconductivity in hydrogen-rich ma- 
terials at high pressures", thesis, Johannes GutenbergUniversitat Mainz, 2016.

[15] Physica C Special Issue, "Superconducting Materials: Conventional, Unconventional and Undetermined. Dedicated to Theodore H. Geballe on the year of his 95th birthday", ed. by J.E. Hirsch, M.B. Maple, F. Marsiglio, Vol. 514, p. 1-444 (2015).

[16] S. Senoussi, P. V. S. S. Sastry, J. V. Yakhmi and I. A. Campbell, "Magnetic hysteresis of superconducting $\mathrm{GdBa}_{2} \mathrm{Cu}_{3} \mathrm{O}_{7}$ down to $1.8 \mathrm{~K}$, J. Phys. Colloques, C82163-C8-2164 (1988).

[17] J. E. Hirsch, "Ferromagnetism in metallic hydrogen", Phys. Lett. A 141, 191-195 (1989); "Superconductivity and hydromagnetism", Physica B 163, 291-298 (1990).

[18] L. Mazov, "Comment on "Conventional superconductivity at 203 kelvin at high pressures in the sulfur hydride system", arXiv:1510.00123 (2015); "Interplay between magnetism and superconductivity in metallic hydrogen and hydrides at high pressure", EPJ Web of Conferences 185, 08003 (2018).

[19] H. Nakao et al, "Superconductivity of Pure $H_{3} S$ Synthesized from Elemental Sulfur and Hydrogen", J. Phys. Soc. Jpn 88, 123701 (2019).

[20] A.P. Drozdov, M. I. Eremets and I. A. Troyan, "Superconductivity above $100 \mathrm{~K}$ in PH3 at high pressures", arXiv:1508.06224 (2015).

[21] A.P. Drozdov et al., 'Superconductivity at $250 \mathrm{~K}$ in lanthanum hydride under high pressures', Nature 569, 528531 (2019).

[22] F. Hong et al, "Superconductivity of Lanthanum Superhydride Investigated Using the Standard Four-Probe Configuration under High Pressures", Chin. Phys. Lett. 37, 107401 (2020).

[23] M. Somayazulu et al., 'Evidence for superconductivity above $260 \mathrm{~K}$ in lanthanum superhydride at megabar pressures', Phys. Rev. Lett. 122, 027001 (2019).

[24] A. D. Grockowiak et al, "Hot Hydride Superconductivity above $550 \mathrm{~K}$ ", arXiv:2006.03004 (2020).

[25] P. P. Kong et al, "Superconductivity up to $243 \mathrm{~K}$ in yttrium hydrides under high pressure", arXiv:1909.10482 (2019).

[26] Y. A. Troyan et al., "Anomalous high-temperature superconductivity in $\mathrm{YH}_{6}$ ", arXiv:1908.01534 (2019).

[27] E. Snider et al, "Superconductivity to 262 kelvin via catalyzed hydrogenation of yttrium at high pressures", arXiv:2012.13627 (2020).

[28] D. V. Semenok et al., "Superconductivity at $161 \mathrm{~K}$ in thorium hydride $\mathrm{ThH}_{10}$ : Synthesis and properties", Materials Today 33, 36-44 (2020).

[29] D. Zhou et al, "Superconducting praseodymium superhydrides", Sci. 6, eaax6849 (2020).

[30] D. V. Semenok et al, "Superconductivity at $253 \mathrm{~K}$ in lanthanum-yttrium ternary hydrides", arXiv:2012.04787 (2020).

[31] W. Chen et al, "High-Temperature Superconductivity in Cerium Superhydrides", arXiv:2101.01315 (2021).

[32] F. Hong et al, "Superconductivity at $70 \mathrm{~K}$ in Tin Hydride SnH $H_{x}$ under High Pressure", arXiv:2101.02846 (2021).

[33] W. Chen et al, "Synthesis of molecular metallic barium superhydride: pseudocubic $\mathrm{BaH}_{12}$, Nature Comm. 12, 273 (2021).

[34] L. Ma et al, "Experimental observation of superconductivity at $215 \mathrm{~K}$ in calcium superhydride at high pressures", arXiv:2103.16282 (2021).
[35] Z. W. Li et al, "Superconductivity above 200K Observed in Superhydrides of Calcium", arXiv:2103.16917 (2021).

[36] X. Huang et al, "High-temperature superconductivity in sulfur hydride evidenced by alternating-current magnetic susceptibility", Nat. Sci. Rev. 6, 713 (2019).

[37] V. Struzhkin et al, "Superconductivity in La and Y hydrides: Remaining questions to experiment and theory", Matter and Radiation at Extremes 5, 028201 (2020).

[38] I. Troyan et al, "Observation of superconductivity in hydrogen sulfide from nuclear resonant scattering", Science 351, 1303 (2016).

[39] J. E. Hirsch, "About the Pressure-Induced Superconducting State of Europium Metal at Low Temperatures", arXiv:2012.07537 (2020), Physica C doi.org/10.1016/j.physc.2020.1353805 (2020).

[40] M. Debessai, T Matsuoka, J J Hamlin, J S Schilling and K Shimizu, "Pressure-Induced Superconducting State of Europium Metal at Low Temperatures", PRL 102, 197002 (2009).

[41] J. Song et al, "Pressure-Induced Superconductivity in Elemental Ytterbium Metal", Phys. Rev. Lett. 121, 037004 (2018).

[42] J. J. Hamlin, "Superconductivity studies at extreme pressure", Washington University, 2007.

[43] D. Semenok and A. R. Oganov, "Measuring the Meissner effect at megabar pressures", Nat. Sci. Rev., Volume 6, 856 (2019).

[44] Y. A. Timofeev et al, "Improved techniques for measurement of superconductivity in diamond anvil cells by magnetic susceptibility", Rev Sci Instrum 73, 371 (2002).

[45] E. Gregoryanz et al, "Superconductivity in the chalcogens up to multimegabar pressures", Phys Rev B 65, 064504 (2002).

[46] S. Deemyad S and J. S. Schilling, "Superconducting phase diagram of Li metal in nearly hydrostatic pressures up to 67 GPa", Phys. Rev. Lett. 91, 167001 (2003).

[47] M. Einaga et al, "Crystal structure of the superconducting phase of sulfur hydride", Nature Physics 12, 835 (2016).

[48] S. A. Solin et al, "Enhanced Room-Temperature Geometric Magnetoresistance in Inhomogeneous NarrowGap Semiconductors", Science 289, 1530 (2000).

[49] T. H. Hewett and F. V. Kusmartsev, "Geometrically enhanced extraordinary magnetoresistance in semiconductor-metal hybrids", Phys. Rev. B 82, 212404 (2010).

[50] See some examples in Y. Kopelevich, R. R. da Silva and B. C. Camargo, "Unstable and elusive superconductors", Physica C 514, 237 (2015).

[51] L. Ma et al, "Experimental Syntheses of Sodalitelike Clathrate EuH6 and EuH9 at Extreme Pressures", arXiv:2002.09900 (2020).

[52] D. V. Semenok et al, "Novel Strongly Correlated Europium Superhydrides", J. Phys. Chem. Lett. 12, 32 (2021) and references therein.

[53] J. Zhang et al, "High-temperature superconductivity in the Ti-H system at high pressures", Phys. Rev. B 101, 134108 (2020).

[54] Y. Ge et al, "Hole-doped room-temperature superconductivity in $H_{3} S_{1-x} Z_{x}(Z=C, S i)$ ", Materials Today Physics 15, 100330 (2020).

[55] S. D. Cataldo et al, " $\mathrm{LaBH}_{8}$ : the first high-Tc lowpressure superhydride", arXiv:2102.11227 (2021).

[56] V. V. Struzhkin, "Superconductivity in compressed 
hydrogen-rich materials: Pressing on hydrogen", Physica C 514, 77 (2015).

[57] Bernd Matthias, a prodigious experimentalist that discovered many new superconducting materials through intuition and empirical reasoning in the period 1953-1980, emphasized throughout his career the inability of BCSEliashberg theory to aid his search for new superconduc- tors.

[58] J. E. Hirsch, "BCS theory of superconductivity: it is time to question its validity", Phys. Scripta 80, 035702 (2009).

[59] J. E. Hirsch, "Superconductivity begins with H", World Scientific, Singapore, 2020. 